\title{
Improving the theory for calculating the rational modes of traction electrical equipment
}

\author{
$A E$ Auhadeev*, $R G$ Idiyatullin, $P$ P Pavlov, $V M$ Butakov, $L N$ Kisneeva and $D I$ Tukhbatullina \\ Kazan State Power Engineering University, Kazan, Krasnoselskaya Street, 51, 420066, Russian Federation
}

\begin{abstract}
Research shows that the existing theory and methods for calculating rational operating modes of traction electrical equipment do not provide a sufficient level of adequacy under real operating conditions of urban electric transport, which is due to the high complexity of the processes and the lack of a sufficiently developed methodology for their interpretation in industry theory. The purpose of the study was researching the process of electric traction as a result of transformation electrical energy in purposeful mechanical movement of electric rolling stock with the implementation of all technological conditions of production and technical system of urban electric transport. The scientific hypothesis that the modes of operation of electric traction equipment as part of an automated electric drive of the rolling stock of urban electric transport are formed under the influence of a complexly organized technological process of electric traction and are the topological basis of its structure is theoretically substantiated. The main result of the research is the formulated theoretical system which allows us to present the process of electric traction as a system consisting of five levels that are in hierarchical relations and solve each specific problem of building the movement of electric transport. Such an approach will contribute to the development of theory and methods for calculating rational operating modes of traction electrical equipment of urban electric transport, the improvement of the energy efficiency of which is the actual scientific problem.
\end{abstract}

\section{Introduction}

For the effectuating of managed by mechanical movement on the modern urban electric transport (UET) is widely used automated traction electric drive, the rising of energy efficiency of which is an actual scientific problem, that has national economic importance. The effectuating of the set operating modes of the traction electrical equipment that is included in the electric drive occurs through an indirect control system (driver controller/driver) in an automated one, and when using an automated driving system - in automatic modes [1 - 3]. In this case, the modes of motion of the electric rolling stock (ERS) during the implementation of electric traction, are determined by the modes of operation of traction electrical equipment (TEE).

The common theory of UET electric equipment, based on the methodological provisions of the theory of electric machines, the theory of automatic control and automated electric drive and the theory of electric traction, has many methods for computation rational modes of operation of the TEE, which as practice shows, do not always show a level of enough adequacy for UET. Obviously, this is connected to the high complication of the processes defining the formation of the operation mode of the TEE during the effectuating of electric traction in the UET system and the absence of a sufficiently detailed methodology for their interpretation in the branch theory $[4,5]$. On this background, many researchers reaffirm that experienced, technically welltrained drivers are able to realize rational modes of operation of the TEE, providing ERS modes of motion that save up to $10 \%$ of electricity compared to the resulting calculations and recommended to the execution of the card of modes [6 - 8]. All this determines the relevance of the scientific problem of the development of the theory and methods of calculating rational operating modes of TEE of UET based on the elaboration of a methodology for describing the processes that define the formation of operating modes of TEE in structure of an automated electric drive when effectuating the technological process of electric traction in the production and technical system UET.

As stated above, questions traction electrical theory related to the calculation of technological modes of operation as an element of the automated electric drive ERS are in the subject field of the theory of electric traction. This fact defines the possibility of developing the theory and methods for calculating rational operating modes of traction electrical equipment by improving the fundamental ideas about the principles of the formation of electrical traction as a process of converting electrical energy into a purposeful mechanical movement of ERS in the effectuating of all technological conditions of the production and technical system of UET. For this, the authors formulated a scientific hypothesis founded on a system approach and synergetic methodology on the interpretation of electric traction as an open complexly

Corresponding author: auhadeevkgma@yandex.ru 
organized process, the topological basis of a multi-level configuration of which is the effectuating of TEE operation modes in composition of an automated electric drive [9 - 12]. Such a methodological approach will help us to develop concepts about the formation of the operating mode of traction electrical equipment, the architecture of interplay with other technical, technological and production processes that characterize the effectuating of electric traction, which will help to solve the actual scientific problem of raising the efficiency of the automated traction drive in the UET system.

\section{Theoretical study materials}

Based on the proposed scientific hypothesis, technological electric traction process, the main task of which is the targeted mechanical motion of the ERS, effectuating by the TEE in the structure of an automated traction drive, in the common case can be represented by a set of hierarchically aligned subprocesses. To describe these subprocesses, it is expedient to use the theory of "movement constructing" suggested by the famous biomechanics of the first half of the twentieth century. N.A. Bernstein [13] and has found applyingin robotics and mechatronics [14]. According to this theory, the implementation of controlled movement of objects or systems of different nature, including biological and technical, is fulfilled by means of its "construction". Wherein building of movement occurs in the common case by 5 levels ("A", "B", "C", "D", "E"), which are called "levels of movement construction" [13]. The theory of movement construction defines the mechanisms of motion control and directing, which are formed in a specific way by levels and have a complex structure. Such a mechanism of "movement constructing" permits to organize an effective algorithm of "constructing solutions" in directing the motion of complex dynamic systems [14].

According to this theory, the levels of building of the ERS motion during the effectuating of electric traction can be identified as follows:

Level $\boldsymbol{A}\left(\mathbf{L}_{\mathbf{A}}\right)$ - a specific level of motions constructing, on which movement as such is missing, but at the same time there are processes related with preparing for motion, i.e. resource provision of the ERS movement process.

Level $B\left(\mathbf{L}_{\mathbf{B}}\right)$ - the level of constructing movements in the own coordinates of system (the special ERS), i.e. dimensional, temporary and power coordination of the mechanical work of the TEE in composition of an automatized traction electric drive.

Level $\boldsymbol{C}\left(\mathbf{L}_{\mathbf{c}}\right)$ - the level of constructing movement in the near objective are, i.e. dimensional, temporary and power coordination of the managed mechanical movement of ERS in conditions of the route of movement (plan and profile of the track, length of the stage, etc.).

Level D $\left(\mathbf{L}_{\mathbf{D}}\right)$ - the level of building of the system's motions when interplaying with objects of the near area, defined by the concepts about their qualities and properties of reciprocal relations (based on objective monitoring of parameters by measuring tools), i.e. effectuating of a focused on the mechanical motion of ERS in conditions of the technology of the transportation process (movement schedule, inter-train interval, running time, etc.)

Level $\boldsymbol{E}\left(\mathbf{L}_{\mathbf{E}}\right)$ - the level of building of the system motion in the interacting with objects of the near area, based on knowledge of their qualities and properties of reciprocal relations, i.e. effectuating of hypothetical ("idealized") mechanical motions of ERS, gratifying the main predictive task parameters of the production process of the UET, formed by the organizational control system.

Levels $\left(\mathbf{L}_{\mathbf{A}}-\mathbf{L}_{\mathbf{E}}\right)$ of construction motion are line up according to the hierarchy of special solvable problems. Herewith, the tasks are solved in parallel, for each level they are permanently formed by the higher level and manage the lower level, obey to the conditions of the task of the common process of EPS motion. In this way, the hierarchy of subprocesses, realizable tasks at each level of movement constructing, going into interconnecting, form the structure of the process of mechanical motion of ERS.

The above process of ERS movement can be conditionally submit as the "mechanical" part of the effectuating of electric traction. It is obvious that there is also an "electrical" part, which defines the transformation processes of electrical energy, the formation of electrical modes of the TEE, cooperation with the environment, the energy system, other ERS, etc. Such processes also have a clear hierarchical structure and, in general, can be submit by 5 levels of the electric traction system constructing [15] (both on direct and alternating currents).

The research of mechanical and electrotechnical components of technological process of the electric traction lets to make the conclusion that the main process is also a system of levels connected to hierarchical subordination and in relationship of effectuating of various special tasks which in total solve the main problem. The real technological process of electric traction cannot be definitely separated into mechanical and electrical parts, but the similarity of their hierarchical structures lets us to formulate the main features of the construction of the electric traction process: multilevel process; levels hierarchy; parallelism and interrelation of subprocesses; interchange of energy, material and information resources between levels and the external environment.

In view of the above features, we formulate a theoretical system (scientific idea) construction levels of the electric traction process. We take as a basis the position of the theory of the building of movement, since the main task of electric thrust is directing mechanical movement of ERS. This theoretical system of hierarchy of levels of the process building of electric traction is graphically presented in Figure 1. Consider its structure and basic principles of construction:

The multilevel process of electric traction is defined by the availability in its structure of some levels (subprocesses), each of which solves a concrete specific 
task, which differs from the others, but in total with others, directed at solving the main task of the process. Such a subprocess or a group of subprocesses can form one of the levels of the system. So in the structure of the process of electric traction it is proposed to separate 5 levels:

The level of resource supply $\left(L_{1}\right)$. By analogy with the level of $\mathbf{L}_{\mathbf{A}}$ movement construction is defining for the whole process of electric traction. At this level, the functional readiness of the process to the action is provided by security a stable and reliable energy and technical (with specified reliability indicators) supply of the transportation process, but the formation of traction does not occur.

The level of implementation of the work of electrical equipment $\left(\mathbf{L}_{2}\right)$. By analogy with the level $\mathbf{L}_{\mathbf{B}}$, at this level is reviewing a subprocess effectuating in the system limited by the object's own coordinates (in this case in the ERS system), excluding the action of outer forces that change the pulse (amount of movement) of the subject (but with the proviso exchange of energy, matter and substance with the outer environment warming and cooling of the TEE, electromagnetic cooperation, moisture changes, etc.). It is conditionally possible to present that the ERS is hung on cables above the rails and, therefore, in relation to ERS external tangent thrust or resistance forces to motion are absent. In this case, the electric traction created by the TEE, is realized in the form of torque attached to the wheel pair or wheel.

The subprocess of this level is characterized by determined algorithms of the TEE work and an indirect managing system, which are defined by the passport electrical and electromechanical characteristics of elements included in them. These algorithms are formed at the engineering phase the ERS and univocal define the effectuating modes of operation, i.e. at the same external conditions, the ERS realizes the same characteristics. This is a feature of this level, is that the algorithms for development electric traction are unchanged and are defined at the engineering phase by using a TEE with specific characteristics. Changing the algorithms of the electric traction effectuation is demand of changing the characteristics of individual elements of the traction electric drive system.

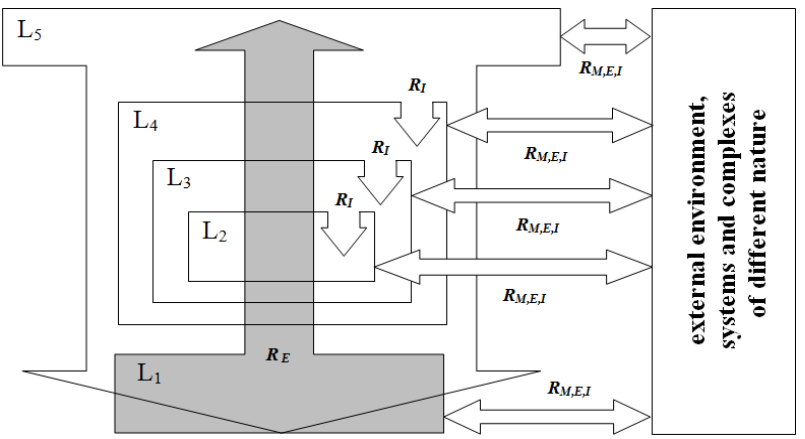

Fig. 1. Theoretical system of construction process of electric traction: $\mathbf{L}_{\mathbf{1}}-\mathbf{L}_{\mathbf{5}}$ - levels of process of electric traction, $\boldsymbol{R}_{\boldsymbol{M}, \boldsymbol{E}, \boldsymbol{I}}-$ exchange of material, energy and information resources respectively.
The level of controlled motion of ERS (L3). At this level, by analogy with $\mathbf{L}_{\mathbf{C}}$ level, a subprocess of cooperation of the ERS with the near area and its elements is formed. So, in the interaction of the wheelset with the elements of the way, there is a tangential thrust force $\mathbf{F}_{\mathbf{K}}$, external to the ERS, leading to motion, and the electrical and electromechanical characteristics of the traction electrical equipment form the ERS traction characteristic. The produced $\mathbf{F}_{\mathbf{K}}$ is managed, that allow to implement the direct motion of the ERS. ERS directed either by the driver or by the automatic driving system. Various parameters of the near space, representing the physical characteristics of the plan and profile of the path, climatic and weather conditions, voltage at the current collector, etc., define the different effectuatings of electric traction.

The distinction between this level and the next one is that in this case electric traction, and, consequently, managed motion is not yet "purposeful", i.e. implements some target, particularly - to transport passengers from one point to another, following in the schedule, in this case the qualifications of drivers at this level are not taken into account.

The level of realization of the technological process UET ( $\left.\mathbf{L}_{4}\right)$. At this level, by analogy with the level of $\mathbf{L}_{\mathbf{D}}$, an interplay subprocess of ERS with the near space and its items is formed with considering their concrete properties and characteristics, for example, the effectuation of the demanded running speed on the stretch considering with the schedule of motion, provision of the speed limits on separate sections, etc. The motion already is not just "controlled", but "purposeful", i.e. directed at the achieving a concrete target - provision the transportation of passengers on the route network according to the technology of the vehicular process of the UET and the quality requirements of train work. At this level, electric traction depends on the professional qualifications level of the driver or the ERS machinist.

The level of realization of the production process UET (L5). This level of the electric traction construction system is abstract and match to the $\mathbf{L}_{\mathbf{E}}$ level in the theory of building movement. Its abstractness is related with the formation of an "idealized" process of electric traction, which lower levels seek to implement. Conditionally, at this level, a "program is built up", by which the electric traction process has to be realized, according to the forecast "idealized" process, which permanently manages this process and predetermines it (for example, the forecast level of electricity intake, passenger traffic, etc.). This subprocess adjust taking into account the strategy of the production process of the transport production UET.

The fact that the subprocesses of the lower levels are part of the subprocesses of higher levels (see. Fig. 1) is the hierarchy of the electric traction process. Processes of higher levels predetermine the parameters and conditions for the effectuating of $\boldsymbol{R}_{\boldsymbol{I}}$ subprocesses of lower, thereby "submitting" them to themselves. Wherein, the higher the level of the system, the more complex the electric traction effectuating task in the UET system, it solves. For example, the subprocess of 
the managed motion of ERS $\left(\mathbf{L}_{\mathbf{3}}\right)$ includes in its composition the subprocess of implementation of the electric equipment operation $\left(\mathbf{L}_{2}\right)$ and wherein is part of the subprocess $\left(\mathbf{L}_{4}\right)$ defined by the technology of the transportation process. In this case, all these subprocesses include a subprocess of reception, transformation and allocation the energy consumed by the EPS (power supply subprocess - $\mathbf{L}_{\mathbf{1}}$ ), which does not form a motion, but which ensures electrical traction for the whole process, and also submit the subprocess of effectuating the strategy of the transport production process (L5). This subprocess is the highest in this hierarchical system, because defines the requirements and conditions for the effectuating of all previous subprocesses.

The parallelism of the subprocesses happening at each level is caused by the mechanism for the effectuating of electric traction and the vertical hierarchy of system levels. Levels of a higher order form their subprocesses not at the end of the subprocess of the lower level of the system, but at its realization moment with continuous interplay, which defines the interconnection of subprocesses. Wherein, the effectuating of their special task the higher level is built on the basis of the lower level task, fully defining the conditions and parameters of its realization (Figure 1). So, the directed motion of ERS $\left(\mathbf{L}_{3}\right)$ on an elementary section of the track (with defined parameters) is lined up on the basis of the effectuating level of the ERS electric equipment work $\left(\mathbf{L}_{2}\right)$, which forms algorithms and modes of the automated ERS electric traction drive operation for the concrete conditions (plan and profile of the specified site, climatic and weather conditions, etc.) in which the subprocess $\mathbf{L}_{\mathbf{3}}$ lines up. When performing the technology of ERS transportation $\left(\mathbf{L}_{4}\right)$, the motion on the route network at compliance the running speed and the inter-train interval in difficult traffic situation is realized on the basis of the subprocess of the ERS managed motion level in the elementary section, forming with considering the ERS train work conditions. Analogously, the building of movement for all levels is performed.

The exchange of energy, material and information resources between the levels and the external environment is one of the main conditions for the realization of the electric traction process in an open complex organized UET system [16, 17]. Conditionally, the structural scheme of the energy exchange $\boldsymbol{R}_{\boldsymbol{E}}$, material $\boldsymbol{R}_{\boldsymbol{M}}$ and information $\boldsymbol{R}_{\boldsymbol{I}}$ resources between the levels of electric traction constructing and the external environment is presented in Figure 1. This condition can be strictly effectuated only in the common case, and in real conditions, as a rule, only some interplays are obvious. So, for example, energy exchanges (electrical, thermal, mechanical energy, etc. [18]), and material resources (including financial and personnel [19]) are obvious. The definition of "information - as measures of Order" is used in the synergetic methodology when describes open complex organized system, in contrast to the concept of "entropy - as a measure of Chaos" [20]. We will understand any interplays leading to the "increased Order" of processes and the system as a whole, by the exchange of information resources. It can be different restrictions, conditions and requirements for the parameters of the processes and phenomena (various for each level (subprocess)), expressed also in the regulations, instructions, norms, etc. i.e. all that limits ("orders") the large multitude ("Chaos") of the effectuating options of a process or its structure.

\section{Conclusion}

As a result of a theoretical research based on the proposed hypothesis, the authors formulated a scientific concept representing a technological process of electric traction, the main task of which is to transform the electrical energy of a source into a purposeful mechanical motion of ERS, an aggregate of hierarchically arrayed subprocesses, each of which is in the hierarchical subordination of a higher level subprocess and forming the conditions for the effectuation of the downstream subprocess, solves a separate specific subtask of motion construction.

The proposed theoretical system will improve the common theory and calculation methods of rational operating modes of TEE of UET by improving the methodological foundations of the principles of electric traction process forming when effectuating the technology of the transportation process in the UET production and technical system. The identified structural and functional connections of process of effectuating of TEE operation, which characterize the interaction architecture with other technical, technological and production processes providing the implementation of electric traction, will determine rational modes and control ways of automated traction electric drive, corresponding to the actual operating conditions of the EPS. It will help to solve the problem of increasing efficiency of electric traction using which is the point of the UET production process management application.

\section{References}

1. V.V Biryukov, E.G. Porsev, Electric Traction Drive, 315 (2018)

2. M.A. Sleptsov, G.P. Dolaberidze, A.V. Prokopovich, The Basics of Electric Transport, 464 (2006)

3. I.S. Efremov, G.V. Kosarev, Theory and calculation of electrical equipment of the rolling stock of urban electric transport, 480 (1976)

4. I.P. Isaev, A.T. Golovaty, Rules of traction calculations need to be revised, Lokomotiv, 8, 6-8 (1992)

5. Ya. Eiduks, Methods of traction calculations need to be specified, Railway of Latvia, 50, 8-9 (1993)

6. S.I. Osipov, E.V. Gorchakov, I.P. Isaev, Rational train driving modes and testing of locomotives, 280 (1984)

7. V.E. Rosenfeld, I.P. Isaev, N.N. Sidorov, Theory of electric traction, 328 (1983) 
8. A.E. Aukhadeev, Modeling of thrust modes of electric power electric transport, 176 (2006)

9. Yu.A. Rylov, A.E. Aukhadeev, A.H. Mukimov, V.I. Karimov, Realization of electric traction in the production and technical system of urban electric transport, Modern scientific research and development, 8(16), 487 -491 (2017)

10. E.M. Hakimov, Dialectic of hierarchy and nonhierarchy in philosophy and scientific knowledge, 288 (2007)

11. Yu.A. Rylov, A.E. Aukhadeev, V.V. Grishina, A.R. Salikhova, R.A. Rashitova, Specificity of electric traction of urban transport, Herald of Contemporary Studies, 11-1(14), 250-253 (2017)

12. A.E. Aukhadeev, R.S. Litvinenko, Yu.A. Rylov, A.G. Hairullin, City electric transport from the standpoint of synergetic methodology, World of Transport and Technological Machines, 1(60), 6773 (2018)

13. N.A. Bernstein, On the construction of movements, 234 (1947)

14. A.K. Platonov, On the construction of motions in ballistics and mechatronics. Applied mechanics and motion control, 127-222 (2010)

15. V.I. Sopov, N.I. Shchurov, Electric power supply systems for direct current electric transport, 728 (2013)

16. G. Haken, trans. from English by V.I. Emelyanova, Eds. Yu.L. Klimontovich, S.M. Osovts, Synergetics, 423 (1980)

17. A.E. Aukhadeev, Self-development of the transport system of the modern city: Search for an innovative model of intellectual management, 220 (2014)

18. V.V. Deev, G.A. Il'in, G. Afonin, Traction trains, 264 (1987)

19. E.M. Khusnutdinova, G.R. Khamidullina, P.P. Pavlov, A.E. Aukhadeyev, R.S. Litvinenko, A.N. Khusnutdinov, Training transport specialists based on a contemporary view of self-organization of sophisticated developing systems, IOP Conference Series: Materials Science and Engineering, 412(1), 012047 (2018)

20. I. Prigozgin, I. Stengers, trans. from English by Yu.A. Danilov, Eds. V.I. Arshinov, Yu.L. Klimontovich, Yu.V. Sagnova, Order from Chaos: A New Dialogue between Man and Nature, 432 (1986) 